\title{
Evaluating the use of an electronic inclinometer in correcting rotational disorders of the hip in children
}

\author{
Ishaan Swarup ${ }^{\mathrm{a}, *}$, Christine Goodbody ${ }^{\mathrm{a}}$, Elizabeth Gausden ${ }^{\mathrm{a}}$, Douglas Mintz ${ }^{\mathrm{b}}$, \\ David Scher ${ }^{\mathrm{a}}$, Roger Widmann ${ }^{\mathrm{a}}$ \\ a Division of Pediatric Orthopaedic Surgery, Hospital for Special Surgery, 535 East 70th Street, New York, NY, 10021, United States \\ b Division of Radiology and Imaging, Hospital for Special Surgery, 535 East 70th Street, New York, NY, 10021, United States
}

\section{A R T I C L E I N F O}

\section{Article history:}

Received 7 March 2018

Accepted 16 June 2018

Available online 19 June 2018

\section{Keywords:}

Rotational disorders

Torsional disorders

Derotation osteotomy

Accuracy

Inclinometer

\begin{abstract}
A B S T R A C T
Background: Femoral derotation osteotomies are commonly performed to correct rotational disorders of the hip in children. Surgical correction is typically assessed visually with the use of a goniometer or osteotomy template, but these methods have been shown to be inaccurate. In this study, we aimed to determine the accuracy of an electronic inclinometer in performing derotation osteotomies of 2 different magnitudes in comparison to commonly used visual techniques.

Methods: We performed derotation osteotomies of $15^{\circ}$ and $30^{\circ}$ in a synthetic femur model using a goniometer, triangle osteotomy template, and electronic inclinometer. The degree of derotation was assessed by computed tomography (CT) and measured by a musculoskeletal radiologist. Statistical analyses included descriptive statistics, as well as t-test, analysis of variance (ANOVA), and Fisher's exact test to determine differences between osteotomy techniques.

Results: We performed 40 proximal femoral derotation osteotomies. The mean difference from the planned derotation was $5.9^{\circ}$ for the goniometer technique, $3.8^{\circ}$ for the osteotomy triangle technique, and $3.2^{\circ}$ for the electronic inclinometer technique $(\mathrm{p}$-value $=0.048)$. There was no difference between the techniques for $15^{\circ}$ derotation osteotomies ( $\mathrm{p}$-value $\left.=0.28\right)$; however, there was a trend towards significance for $30^{\circ}$ derotation osteotomies $(p$-value $=0.07)$. Fewer osteotomies had a difference greater than $3^{\circ}$ from the planned derotation using the electronic inclinometer technique ( $\mathrm{p}$-value $=0.04$ ). Conclusions: The accuracy of derotation osteotomies can be improved with the use of an electronic inclinometer. This technology has the potential to improve surgical technique, minimize surgical error, and possibly improve patient outcomes.
\end{abstract}

\section{Introduction}

Rotational abnormalities are found in approximately 5\% of the population, ${ }^{1}$ and rotational disorders are commonly treated by pediatric orthopedic surgeons. These deformities can arise from the femur, tibia, or both, and they may be associated with several underlying conditions including disorders of the hip and neuromuscular conditions such as cerebral palsy. ${ }^{2}$ The location of the pathology can be determined by a patient's rotational profile on physical exam, and it can be further quantified with the use of gait analysis or advanced imaging, such as computed tomography (CT). When rotational disorders arise from the femur, they result in abnormalities in hip rotation and femoral anteversion. ${ }^{3}$ Rotational abnormalities of the hip are often associated with cosmetic

\footnotetext{
* Corresponding author.

E-mail address: swarupi@hss.edu (I. Swarup).
}

deformity and gait abnormalities, sometimes resulting in functional impairment and pain. Some studies have also suggested that excessive femoral anteversion may be associated with an increased risk of degenerative joint disease. ${ }^{3}$

The surgical management of hip rotational abnormalities consists of derotation osteotomy. Typically, k-wires are placed proximal and distal to the osteotomy site as a fixed reference, and after derotation, the angle between them is measured to determine the degree of correction. Intra-operatively, the correction is assessed visually with the use of a goniometer or osteotomy template. These techniques are user-dependent, and they are susceptible to inaccuracy and error. ${ }^{2,4-7}$ Over-correction and under-correction have been reported in the literature with error rates as high as $10 \%{ }^{8}$ Furthermore, it is known that measured rotation at the time of surgery differs from post-operative radiographic measurements in up to $50 \%$ of cases. ${ }^{8,9}$ These findings suggest that current methods for measuring derotation intraoperatively are suboptimal, which may ultimately result in 
persistent or additional symptoms, re-operation, and increased patient morbidity. 8,10

Inclinometers and smart phone inclinometer applications have been recently used clinically in an attempt improve accuracy. ${ }^{7}$ Traditionally, electronic inclinometers have been used by carpenters, surveyors, and engineers to assess tilt or slope. Electronic inclinometers have demonstrated accuracy in measuring static rotational differences as well as improving accuracy in total knee arthroplasty. ${ }^{7,11}$ However, there have been no studies to our knowledge assessing the use of an electronic inclinometer in performing derotational osteotomies. This study was designed to assess the accuracy of femoral derotation osteotomies performed using an electronic inclinometer, compared to commonly used visual techniques. We hypothesize that derotation osteotomies using an electronic inclinometer will be more accurate than osteotomies using commonly used visual techniques. The use of inclinometer technology may allow for more accurate and precise measurement of correction, and may improve upon traditional visual techniques.

\section{Methods}

The aims of this study were to assess the accuracy of an electronic inclinometer in performing femoral derotation osteotomies, as well as to compare this method to commonly used visual techniques. In order to accomplish these aims, we performed multiple derotational osteotomies of $15^{\circ}$ and $30^{\circ}$ in a synthetic femur model using an electronic inclinometer, a goniometer, or a triangle osteotomy template. Pediatric sawbone femurs were used as an analogue to pediatric long bones. Standardized pediatric sawbones were purchased from a reputable vendor (Sawbones, Vashon Island, WA), and a commercially available electronic inclinometer (Hammerhead ${ }^{\mathrm{TM}}$ ) was used for all osteotomies (Fig. 1A). This inclinometer has a slotted base which allows it to be positioned in-line with k-wires even in the presence of a softtissue envelope, and it has a reported accuracy of $\pm 0.1^{\circ}$. Large diameter k-wires $(2 \mathrm{~mm})$ were used to minimize the risk of bending the wires when using the slotted electronic inclinometer. In addition, a standard goniometer (Fig. 1B) as well as $15^{\circ}$ and $30^{\circ}$ triangle osteotomy templates were used in this study (Fig. 1C).

\subsection{Osteotomy technique}

The same surgical team performed all osteotomies, and this team consisted of two fellowship-trained pediatric orthopaedic surgeons and two orthopaedic surgery residents. Derotation osteotomies were performed in the proximal femur (subtrochanteric) since osteotomies in this region are technically challenging and associated with more errors in correction. ${ }^{8}$ We employed an osteotomy technique that is well described and commonly used at our institution. ${ }^{12}$ After placing parallel k-wires proximal and distal to the osteotomy site as a fixed reference (Fig. 2A), a baseline CT

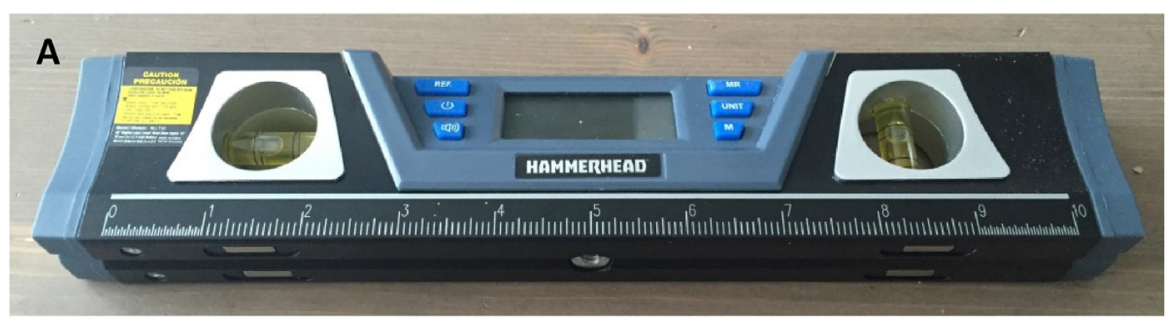

B
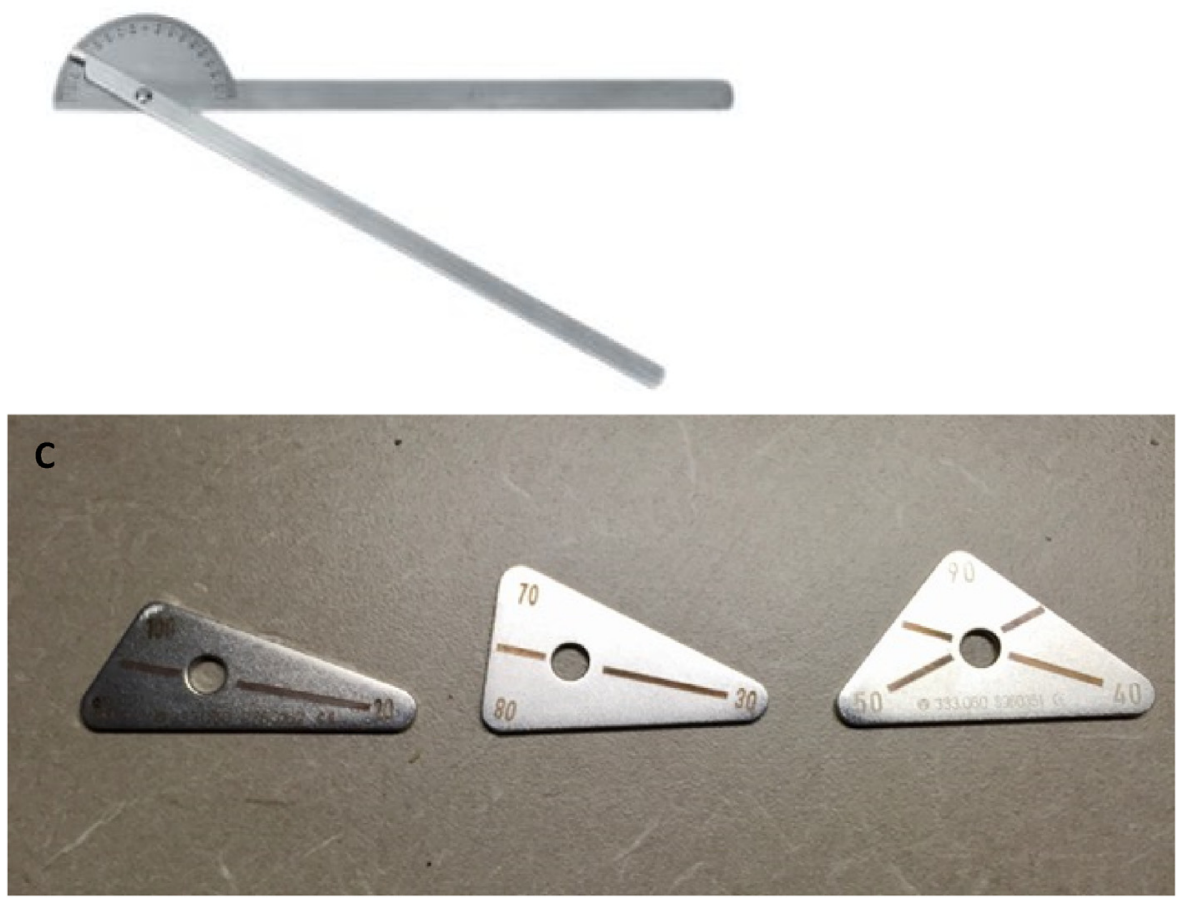

Fig. 1. Examples of an electronic inclinometer with slotted base (A), goniometer (B), triangle osteotomy templates (C). 

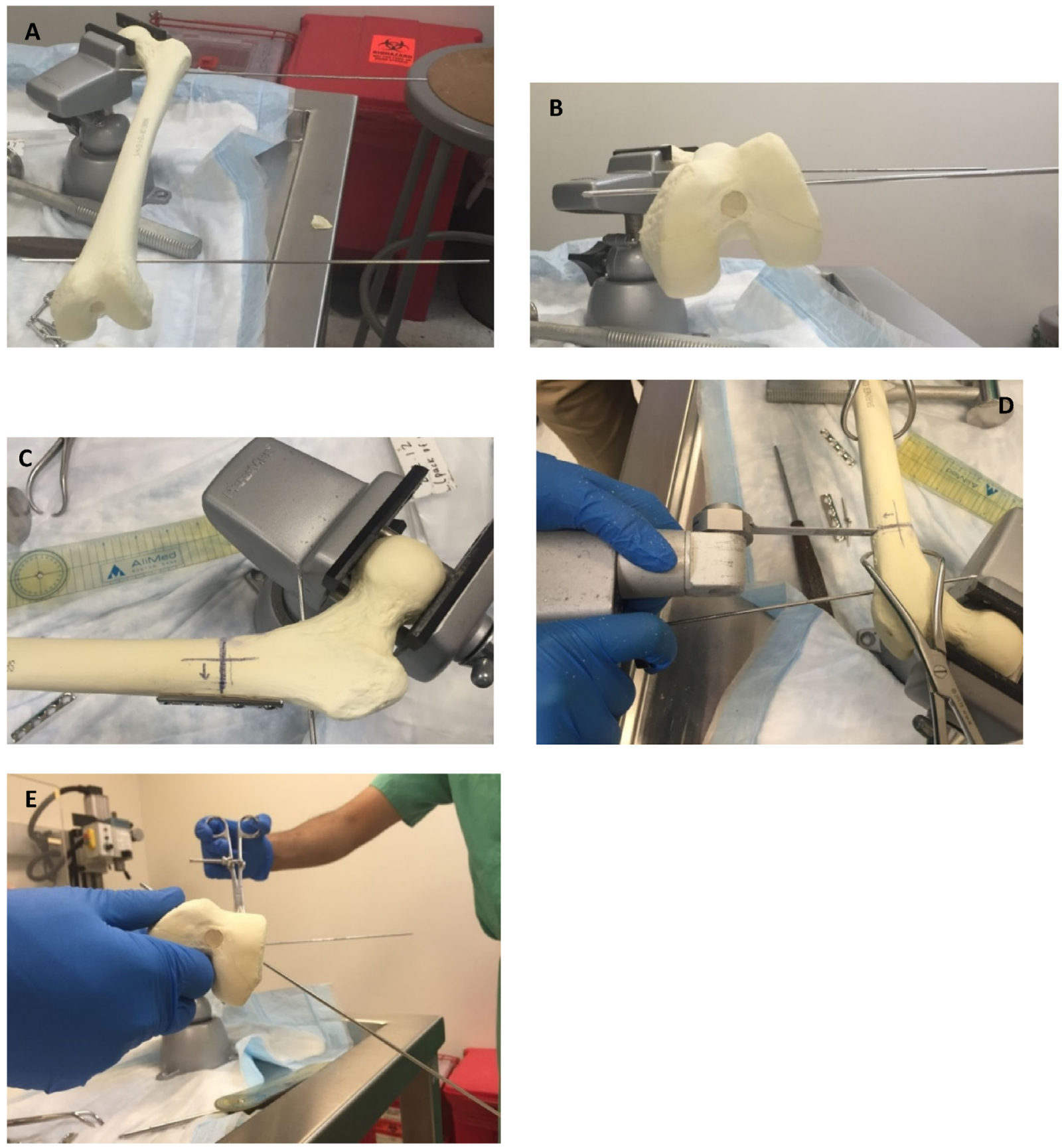

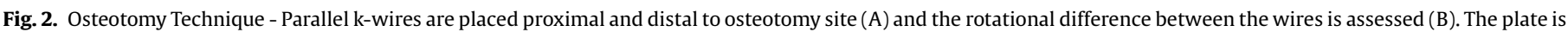

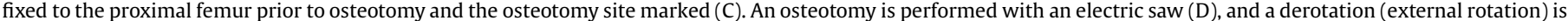
performed with the use of an electronic inclinometer, goniometer, or triangle osteotomy template.

scan was performed to assess the rotational difference between the proximal and distal wires (Fig. 2B). The surgical team was blinded to these baseline radiographic differences. Before the osteotomy, the proximal screw holes were drilled to orient the plate on the proximal femur (Fig. 2C). The plate was then removed, and an electric saw blade was used to perform the proximal femoral osteotomy (Fig. 2D). Derotation of either $15^{\circ}$ or $30^{\circ}$ was performed, and was assessed by using an electronic inclinometer, goniometer, or triangle osteotomy template to determine the rotational difference between the k-wires (Fig. 2E). We alternated between the different techniques in order to minimize the influence of practice effects. The osteotomy was then fixed with a four-hole small fragment LC-DCP plate and four $26 \mathrm{~mm}$ cortical screws (DePuy Synthes, Warsaw, IN). A post-osteotomy CT scan was subsequently performed to assess the rotational difference between the proximal and distal k-wires (degree of derotation).

\subsection{Derotation measurement}

An attending musculoskeletal radiologist blinded to the planned derotation performed all baseline and post-osteotomy measurements. Axial CT cuts were used to determine the radiographic angle between the proximal and distal k-wires. The accuracy of derotation was determined by comparing the planned derotation with the measured radiographic derotation. The difference between planned derotation and measured derotation was recorded as negative (-) if there was under-rotation or positive $(+)$ if there was over-rotation. 


\subsection{Pilot study and power analysis}

The number of sawbones in each group was determined by a pilot study of 10 sawbones using the goniometer technique, which is the current standard of care at our institution. We performed 10 derotation osteotomies using this method $\left(15^{\circ}\right.$ derotation $\times 5,30^{\circ}$ derotation $\times 5$ ), and then calculated the mean difference and standard deviation. The mean difference was defined as the difference between the planned derotation and measured or radiographic derotation, and the mean difference in the pilot study was $5.19^{\circ}\left(\mathrm{SD}: 3.47^{\circ}\right)$.

While there is no consensus on the level of clinically significant under- or over-correction, previous studies have shown that derotation errors of $5^{\circ}$ occur in over half of all derotation cases. ${ }^{7-9}$ As such, we defined $5^{\circ}$ as our effect size for this study. A power analysis was performed to calculate the final sample size for each group using 90\% power (beta) and level of significance of 0.05 (alpha). Based on this power analysis, we needed 5 sawbones per group to detect a difference of $5^{\circ}$ between the goniometer technique and alternative techniques. As a result, our minimum sample size was 30 sawbones ( 5 sawbones/group $\mathrm{x} 3$ techniques $\mathrm{x}$ 2 derotation osteotomy sizes $=30$ sawbones $)$.

\subsection{Statistical analysis}

Statistical analyses included descriptive statistics for accuracy of correction (mean difference from planned derotation), as well as t-test, analysis of variance (ANOVA), and Fisher's exact test to determine differences between osteotomy techniques. We analyzed the data cumulatively for each technique, and also stratified the analysis by the degree of derotation $\left(15^{\circ}\right.$ or $\left.30^{\circ}\right)$.

\section{Results}

In total, we performed 40 proximal femoral derotation osteotomies. We performed 20 osteotomies using a goniometer, 10 osteotomies using a triangle osteotomy template, and 10 osteotomies using an electronic inclinometer. We under-rotated 21/40 sawbone femurs, and over-rotated 19/40 sawbone femurs. The mean difference from the planned derotation was $5.9^{\circ}$ (SD: $3.1^{\circ}$ ) for the goniometer technique, $3.8^{\circ}\left(\mathrm{SD}: 2.1^{\circ}\right.$ ) for the osteotomy triangle technique, and $3.2^{\circ}\left(\mathrm{SD}: 3.3^{\circ}\right)$ for the electronic inclinometer technique $(\mathrm{p}$-value $=0.048)($ Table 1$)$. The difference was greater than $3^{\circ}$ from planned derotation for $17 / 20$ osteotomies using the goniometer technique; 6/10 osteotomies using the osteotomy triangle technique; and 4/10 osteotomies using the electronic inclinometer technique $(\mathrm{p}$-value $=0.04)$ (Fig. 3$)$. No differences were observed when individually comparing the goniometer technique to osteotomy triangle technique ( $\mathrm{p}$-val$\mathrm{ue}=0.23$ ); however, there was a trend towards significance when comparing the goniometer technique to electronic inclinometer technique $(\mathrm{p}$-value $=0.07)$.

\section{1. $15^{\circ}$ Derotation osteotomies}

We performed 20 proximal femoral derotation osteotomies of $15^{\circ}$. In this group, 10 osteotomies were performed using a goniometer, 5 osteotomies were performed using a triangle osteotomy template, and 5 osteotomies were performed using an electronic inclinometer. The mean difference from the planned derotation was $5.6^{\circ}\left(\mathrm{SD}: 2.9^{\circ}\right)$ for the goniometer technique, $2.8^{\circ}$ (SD: $1.8^{\circ}$ ) for the osteotomy triangle technique, and $4.2^{\circ}\left(\mathrm{SD}: 4^{\circ}\right)$ for the electronic inclinometer technique $(\mathrm{p}$-value $=0.28)($ Table 1$)$.

\section{2. $30^{\circ}$ Derotation osteotomies}

We performed 20 proximal femoral derotation osteotomies of $30^{\circ}$. In this group, 10 osteotomies were performed using a goniometer, 5 osteotomies were performed using a triangle osteotomy template, and 5 osteotomies were performed using an electronic inclinometer. The mean difference from the planned derotation was $6.2^{\circ}\left(\mathrm{SD}: 3.5^{\circ}\right)$ for the goniometer technique, $4.8^{\circ}$ (SD: $2.1^{\circ}$ ) for the osteotomy triangle technique, and $2.2^{\circ}$ (SD: $2.3^{\circ}$ ) for the electronic inclinometer technique $(\mathrm{p}$-value $=0.07)($ Table 1$)$.

\section{Discussion}

Femoral derotation osteotomies are commonly performed for hip rotational abnormalities in children. Surgical correction is typically assessed visually with the use of a goniometer or osteotomy template; however these visual techniques can be inaccurate and errors may result in persistent symptoms, additional abnormalities, re-operation, and significant morbidity. ${ }^{2,4-7}$ Electronic inclinometers have been shown to measure rotation more accurately, ${ }^{7}$ but there is a paucity of data on the surgical application of this technology for rotational disorders. In our study, the accuracy of femoral derotation osteotomies was significantly different based on technique, and the greatest accuracy was achieved with the use of an electronic inclinometer. This finding was most notable for osteotomies of $30^{\circ}$ with a trend towards statistical significance. Individual comparison between our current standard technique (goniometer) and the electronic inclinometer technique also revealed a trend towards statistical significance. In addition, there were fewer errors greater than $3^{\circ}$ using the elecontronic inclinometer technique.

Table 1

Mean difference from planned derotation osteotomy.

\begin{tabular}{|c|c|c|c|c|}
\hline & $\begin{array}{l}\text { Number of } \\
\text { Sawbones (N) }\end{array}$ & $\begin{array}{l}\text { Mean Difference From } \\
\text { Planned Derotation }\left({ }^{\circ}\right)\end{array}$ & $\begin{array}{l}\text { Standard } \\
\text { Deviation }\left({ }^{\circ}\right)\end{array}$ & P-Value \\
\hline All Osteotomies & & & & 0.048 \\
\hline Goniometer & 20 & 5.88 & 3.14 & \\
\hline Triangle Template & 10 & 3.80 & 2.14 & \\
\hline Electronic Inclinometer & 10 & 3.21 & 3.26 & \\
\hline 15으 Derotation Osteotomy & & & & 0.28 \\
\hline Goniometer & 10 & 5.55 & 2.95 & \\
\hline Triangle Template & 5 & 2.82 & 1.85 & \\
\hline Electronic Inclinometer & 5 & 4.20 & 4.04 & \\
\hline 30 ㅇ Derotation Osteotomy & & & & 0.07 \\
\hline Goniometer & 10 & 6.21 & 3.46 & \\
\hline Triangle Template & 5 & 4.78 & 2.12 & \\
\hline Electronic Inclinometer & 5 & 2.22 & 2.28 & \\
\hline
\end{tabular}




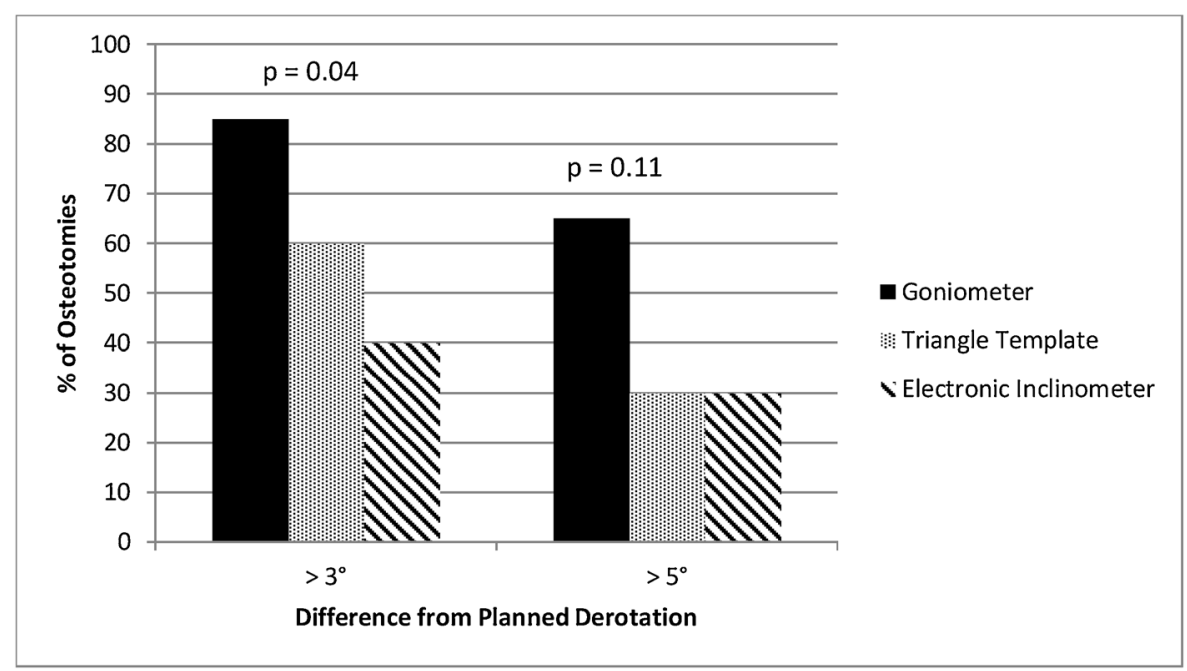

Fig. 3. Proportion of osteotomies with differences greater than $3^{\circ}$ and $5^{\circ}$ from planned derotation.

Graham et al., previously showed that a smart phone inclinometer more accurately measured static differences compared to visual estimation and osteotomy templates, and they advocated for the use of inclinometer technology. ${ }^{7}$ More specifically, these authors used a specific smart phone application, and they assessed four static measurements ranging from $10^{\circ}$ to $60^{\circ}$. In comparison, we used a commercially available electronic inclinometer with a slotted base for k-wires; we performed an assessment of baseline and postosteotomy rotational differences; and we used a standardized osteotomy technique that mimics surgical practice. In addition, our study quantifies the improvement in accuracy that can be expected with the use of an electronic inclinometer compared to common visual techniques.

Despite statistical significance, the clinical significance of our findings is unclear. In general, our findings show that the electronic inclinometer technique is more accurate than the goniometer technique, consistent with the findings of Graham et al. ${ }^{7}$ However, the magnitude of improvement is modest. Further study is needed to determine the clinical significance of improvements in femoral rotation, and our findings suggest that additional improvements in design and technology are needed to further improve the accuracy of derotation osteotomies. While there is no known minimal clinically important difference for derotation osteotomies, ${ }^{7}$ we know that errors in derotation are common and even small errors may have significant implications to functional outcomes and patient disability, ${ }^{8-10,13}$ In the absence of evidence-based guidelines, every effort should be made to minimize error in surgical correction, consistent with standard surgical practice in other subspecialties such as trauma and arthroplasty.

The use of technology has revolutionized surgical practice, and there is an increasing emphasis on the use of technology in orthopaedic surgery. ${ }^{14,15}$ More recently, greater attention is being placed on handheld devices to improve surgical practice and outcomes, ${ }^{16}$ and market research suggests that over 70\% of US physicians use smart phones in clinical practice with this number projected to increase over time. ${ }^{17}$ Inclinometer technology has been used to improve clinical assessment $^{18}$ and surgical practice, ${ }^{11,19}$ and our results suggest that inclinometer technology can be applied to improving the accuracy of derotation osteotomies, which will minimize surgical error and possibly improve patient outcomes after surgery. In the future, this technology may also be adapted and used to address rotational deformities in other long bones, as well as correct coronal plane disorders, sagittal plane disorders, and fracture malunions.

Our study has several strengths. Most notably, we used a robust study design that included a power analysis based on pilot data to determine sample size. We also used standardized surgical technique in order to minimize bias, variability, and practice effects. Similarly, we designed our experiments to mimic surgical practice, including the use of realistic modes of fixation to simulate surgical application and maintain derotation, as well as the use of a slotted electronic inclinometer and large-diameter k-wires. In addition, we performed osteotomies of two different magnitudes to determine any differences in accuracy, and we assessed the magnitude of derotation or error using CT measurements.

In comparison, our study has several weaknesses as well. For instance, all radiographic measurements were performed by a single musculoskeletal radiologist. To address this concern, all CT measurements were repeated by a blinded senior orthopaedic surgery resident, and the inter-observer reliability was found to be similar to other reports in the literature. ${ }^{20}$ While the reliability and reproducibility of $\mathrm{CT}$ assessment is controversial, it continues to remain the gold standard to assess rotational abnormalities. ${ }^{20}$ Furthermore, the electronic inclinometer used in this study is intended for commercial use, and it is not available for intraoperative use. However, the purpose of our study was to investigate the accuracy of inclinometer technology in performing derotation osteotomies compared to common visual techniques. More practically, commercially-available electronic inclinometers placed in sterile bags can be readily used intraoperatively. Lastly, even though osteotomies performed with the triangle osteotomy technique demonstrated similar accuracy to the electronic inclinometer technique, we cannot specifically compare these two techniques. This study was powered to compare our current standard of care (goniometer technique) to alternative techniques, and an adequately powered study is needed to specifically compare the template osteotomy and electronic inclinometer techniques.

In conclusion, our findings support the use of an electronic inclinometer to improve the accuracy of derotation osteotomies, particularly corrections of larger magnitude. Additional study is needed to assess whether improvements in accuracy with the electronic inclinometer can be appreciated at even larger magnitudes of correction. Modifications in the design and technology of an electronic inclinometer would be useful to further improve the accuracy of this instrument and allow for optimal adaptation to intraoperative use. As a whole, electronic inclinometer technology offers an innovative, simple, and costeffective means to improve surgical technique, minimize surgical error, and potentially improve patient outcomes. 


\section{Conflict of interest}

None.

\section{Funding}

This study was partly funded through an institutional research grant. Implants were provided by DePuy Synthes (DePuy Synthes Scientific Research Grant).

\section{References}

1. Torsion of the Lower Limb in Children. Epocrates. BMJ. 2016;(April) n.d. Web. 27.

2. Mylle J, Lammens J, Fabry G. Derotation osteotomy to correct rotational deformities of the lower extremities in children. A comparison of three methods. Acta Orthop Belg. 1993;59:287-292.

3. Schoenecker P, Rich M. The lower extremity. In: Morrissey R, Weinstein S, eds. Lovell E' winter's pediatric orthopaedics. Lippincott Williams \& Wilkins; 2006:1158-1165.

4. Molony DC, Sparkes J, Noonan J, McCormack D. The orthopaedic angle on angles: the accuracy of health care professionals in assessing angular displacement. Eur J Orthop Surg Traumatol. 2004;14:80-83.

5. Moran KM, Ness K, Finkbeiner AA. Visual estimation of angles by orthopedic surgeons. Am J Orthop. 2000;29:361-362 discussion.

6. Rose V, Nduka CC, Pereira JA, Pickford MA, Belcher HJ. Visual estimation of finger angles: do we need goniometers? J Hand Surg [Br]. 2002;27:382384.

7. Graham D, Suzuki A, Reitz C, Saxena A, Kuo J, Testworth K. Measurement of rotational deformity: using a smartphone application is more accurate than conventional methods. ANZ J Surg. 2013;83(December 12):937-941.
8. Kay RM, Rethlefsen SA, Hale JM, Skaggs DL, Tolo VT. Comparison of proximal and distal rotational femoral osteotomy in children with cerebral palsy. $J$ Pediatr Orthop. 2003;23(March-April 2):150-154.

9. Svenningsen S, Apalset K, Terjensen T, Anda S. Osteotomy for femoral anteversion: complciations in 95 children. Acta Orthop Scand. 1989;60(August 4):401-405.

10. Payne LZ, DeLuca PA. Intertrochanteric versus supracondylar osteotomy for severe femoral anteversion. J Pediatr Orthop. 1994;14(January-February 1):39-44.

11. Nam D, Cody EA, Nguyen JT, Figgie MP, Mayman DJ. Extrameduallary guides versus portable, accelerometer-based navigation for tibial alignment in total knee arthroplasty: a randomized, controlled trial. J Arthroplasty. 2014;29 (February 2):288-294.

12. Mylle J, Lammens G, Fabry G. Derotation osteotomy to correct rotational deformities of the lower extremities in children. A comparison of three methods. Acta Orthop Belg. 1993;59(3):287-292.

13. Naqvi G, Stohr K, Rehm A. Proximal femoral derotation osteotomy for idiopathic excessive femoral anteversion and intoeing gait. SICOT J. 2017;3:49.

14. Qureshi S, Lu Y, McAnany S, Baird E. Three-dimensional intraoperative imaging modalities in orthopaedic surgery: a narrative review. J Am Acad Orthop Surg. 2014;22(December 12):800-80910.5435/JAAOS-22-12-800.

15. Zheng G, Nolte LP. Computer-assisted orthopedic surgery: current State and future perspective. Front Surg. 2015;23(December 2):66.

16. Mayman D. Handheld navigation in total knee arthroplasty. Orthop Clin North Am. 2014;45(April 2):185-190.

17. Kiser K. 25 ways to use your smartphone. Physicians share their favorite uses and apps. Minn Med. 2011;94:22-29.

18. Zatt MT, Bateman GR, Adam CJ. Evaluation of the iPhone with an acrylic sleeve versus the Scoliometer for rib hump measurement in scoliosis. Scoliosis. 2012;7:14.

19. Peters FM, Greeff R, Goldstein N, Frey CT. Improving acetabular cup orientation in total hip arthroplasty by using smartphone technology. J Arthroplasty. 2012;27:1324-1330.

20. Yoon RS, Koerner JD, Patel NM, Sirkin MS, Reilly MC, Liporace FA. Impact of specialty and level of training on CT measurement of femoral version: an interobserver agreement analysis. J Orthop Traumatol. 2013;14(December 4):277-281. 